\title{
A Novel Architectural Framework and Configuration Scheme for Spectrum Sensing
}

\author{
Abdoulaye Bagayoko, Dorin Panaitopol, Philippe Delahaye and Christian Mouton \\ NEC Technologies (UK) Ltd., FMDC Lab., ComTech Department, \\ 55 Avenue des Champs Pierreux, Immeuble le Capitole, 92012, Nanterre Cedex, France \\ Email: \{Abdoulaye.Bagayoko, Dorin.Panaitopol, Philippe.Delahaye, Christian.Mouton\}@nectech.fr
}

\begin{abstract}
This paper proposes a novel architecture and configuration scheme for single-node spectrum sensing. It is assumed that the sensing node may use more than one sensing algorithms. The proposed sensing architecture allows a dynamic algorithm selection that depends on the sensing configuration parameters, and it includes a regulatory conformance checking. As a result, local sensing is enhanced by dynamic selection of the most reliable sensing algorithm, while a regulatory conformance indicator shows whether the current sensing result should be assumed reliable or not. Such information could be used further by a cooperative sensing system for efficient data fusion and decision making.
\end{abstract}

\section{INTRODUCTION}

Tremendous changes are occurring in wireless communications so that the mobile phone is rapidly turning into a sophisticated mobile device capable of supporting high computational load applications. The market of smart phones has outpaced the rest of the mobile phone market for several years. Investigations show that the mobile data traffic footprint of a single mobile subscriber in 2015 could very conceivably be 450 times higher from what it was in 2005 [2], and by 2014, almost $66 \%$ of the world's mobile data traffic will be video [3]. These changes come along with a strong demand in bandwidth and high data rate. For example, the data rates provided by the initial High Speed Downlink Packet Access (HSDPA), an extension to $3 \mathrm{G}$ networks, enable a user to access the Internet at speeds up to $1.8 \mathrm{Mbps}$. Enhancements in HSDPA modulation schemes increase this speed up to $10 \mathrm{Mbps}$. With the Long Term Evolution (LTE) technology, we are expecting a peak data rate of $100 \mathrm{Mbps}$ down link/ $50 \mathrm{Mbps}$ up link within $20 \mathrm{MHz}$ bandwidth. With higher modulation and coding schemes, one is already close to the limit of what modulation and coding can bring to data rate enhancement. Therefore, there is a need of better frequency re-use and interference management.

Emerging technologies, including Cognitive Radio (CR) and Software Defined Radio (SDR) [1], are overcoming the problem of spectrum shortage by providing sophisticated techniques to reuse licensed spectrum. A CR uses intelligent signal processing at the physical layer in order to adapt to its environment, to its user's requirements and to the requirements of other radio users sharing the spectrum [4]. CR could therefore provide means to efficiently use the electromagnetic spectrum by detecting and exploiting empty parts (spectrum holes), or by intelligently sharing spectrum with other users (e.g., meeting given interference constraints).

When employed as a secondary user (i.e., non-licensed user), a CR has lower priority in using the spectrum allocated to a primary user (i.e., licensed user). Therefore, a fundamental requirement is to avoid interference with the primary systems in their vicinity. Besides, there is no requirement for primary user networks to change their infrastructure in spectrum sharing with cognitive networks. Therefore, a CR should be able to independently detect primary user presence [6]. To enable such an opportunistic access to the licensed spectrum, different access methods have been proposed. Sensing-based access, where a CR transmits if it finds the licensed band is available, is currently being investigated due to its low deployment cost and its compatibility with the licensed systems [10]. Several techniques, with different characteristics and performances, used to sense a licensed spectrum have been investigated [11][16]. Various aspects and classes of sensing algorithms are presented in [8]. In [7] an architecture and an approach for finding spectrum holes have been proposed. In particular, [7] presents a framework for selecting among various spectrum monitoring methods, taking into account the operational environment, the underlying policies and the sensing methods capabilities. However, the selection criteria in [7] are vague and do not cover some special cases. For example, the cyclostationary detector is more reliable than the energy detector for certain SNR and for given noise estimation performance, but the energy detector could be more reliable than the cyclostationary detector when the noise is accurately estimated. The method in [7] does not cover such specificities. Moreover, no other work, in our best knowledge has never proposed a sensing framework that takes into account the regulatory conformance with the sensing results.

This paper therefore proposes a new architecture and configuration scheme for spectrum sensing. The specificities of this work compared with the state of the art are as follows.

- For the first time, in this paper the authors propose a framework for checking the regulatory conformance with regard to the sensing reliability.

- A knowledge base is proposed to be stored in the sensing nodes. This knowledge base is fed off-line and allows to determine, depending on the values of the sensing configuration parameters, the best sensing algorithm in terms of the mean detection probability. The sensing 
configuration parameters include the frequency band to be sensed, the type of primary signal to be detected, the sensing duration, the maximum allowed false alarm probability, the minimum allowed detection probability, the time available for noise estimation.

- A new architecture for single node spectrum sensing that includes all the above functionalities is proposed.

The goal of this work is therefore to optimize local sensing and to provide a regulatory conformance indicator. As a result, processing sensing results is helped by the regulatory conformance indicator and the overall sensing performance is enhanced.

The remainder of this paper is organized as follows. Section II presents sensing generalities and an overview of spectrum sensing algorithms. Section III introduces a novel architectural framework for sensing configuration using a selection between various sensing algorithms while checking the regulatory conformance. An example is further discussed in Section IV. Finally, conclusions are given in Section V.

\section{Sensing Basics And Overview of SEnsing TECHNIQUES}

In this section we remind some basics of spectrum sensing and we provide a non-exhaustive overview of spectrum sensing techniques.

\section{A. Sensing Basics}

We consider a CR attempting to access a licensed spectrum where a primary user transmits. The CR is assumed to have various sensing capabilities in order to autonomously find possible spectrum holes that can be used for opportunistic communications. The $\mathrm{CR}$ continuous time received signal is

$$
y(t)=h s(t)+w(t),
$$

where $y(t)$ represents the received signal, $h$ is the channel gain from the primary transmitter to the cognitive receiver, $s(t)$ is the primary user signal that the cognitive device has to detect and $w(t)$ is the Additive White Gaussian Noise (AWGN) with variance $\sigma^{2}$. It is assume that the signal is obtained from a specific frequency band with central frequency $f_{c}$ and bandwidth $W$. The signal is sampled at a sampling rate $f_{s}$, where $f_{s} \geq W$. The sampling period is defined as $T_{\text {samp }}=1 / f_{s}$. Then, we can rewrite the different signals as $y(n)=y\left(n T_{\text {samp }}\right), s(n)=s\left(n T_{\text {samp }}\right)$ and $w(n)=$ $w\left(n T_{\text {samp }}\right)$. And it follows that $y(n)=h s(n)+w(n)$. The goal of spectrum sensing is to detect whether there is a primary signal present or not. That is, we want to discriminate between the following two hypotheses:

$$
\begin{array}{ll}
\mathcal{H}_{0}: & y(n)=w(n) \\
\mathcal{H}_{1}: & y(n)=h s(n)+w(n) .
\end{array}
$$

The ideal sensing algorithm should select hypothesis $\mathcal{H}_{1}$ when a primary signal is present, and should select hypothesis $\mathcal{H}_{0}$ otherwise. In spectrum sensing, in general, the detection decision is obtained by comparing a test statistic, which is calculated from the signal samples, with a threshold. If the received signal contains only noise, the test statistic should be lower than the threshold. Otherwise, the test statistic should therefore be higher than the threshold. Therefore, the performance of a sensing algorithm can be expressed in terms of detection probability and false alarm probability. The detection probability represents the probability that the test statistic is higher than the threshold when a primary signal is really present. The false alarm probability represents the probability that the test statistic is higher than the threshold when there is no primary signal present.

\section{B. An Overview of Sensing Algorithms}

In this part, we give a non-exhaustive overview of wellknown sensing techniques.

If the characteristics of the primary signal are known, the optimal detector in stationary Gaussian noise is a matched filter followed by a threshold test [21]. Matched filter detection should require dedicated circuitry to achieve synchrony with the primary system. Therefore, this detection technique may be interesting for early cognitive radio deployments where the secondary system should operate, opportunistically, in a few licensed bands [10]. As the number of wireless applications increases, more licensed bands should be opened for opportunistic access in the future. Therefore, matched filter detection would no longer be viable as its implementation cost and complexity should increase prohibitively with the use of various dedicated circuitries to achieve synchrony with the different primary systems. Sensing techniques requiring less information on the primary signal are more promising in the presence of various primary systems. The energy detection does not require any knowledge of primary signal characteristics [11]. Therefore, the energy detector is a blind detector. However, it is well known that the energy detector is sensitive to the noise uncertainty, [18], that makes tough the use of this technique. When some patterns (e.g., midambles, regularly transmitted pilots, spreading sequences etc.) of the signal are known, waveform-based sensing can be used. In this case, sensing can be performed by correlating the received signal with a known copy, [8]. A cyclostationary-based detection, [12], requires the knowledge of the cyclostationary features of the signal. The cyclostationary detectors are known to be robust in the presence of noise uncertainty [15]. The twostage detector [16], is designed to benefit from the advantages of both the energy detector and the cyclostationary detector. This detection scheme increases the detection probability comparing to a single detector, but it also increases the false alarm probability. Furthermore, in order to reduce the complexity of the GLRT (Generalized Likelihood Ratio Test) cyclostationary detector from [12], the MCAS (Maximum Cyclic Autocorrelation Selection) cyclostationary detector has been proposed in [15]. Radio identification based sensing (see [9] and references therein) can be used to know about the transmission technologies (e.g., Bluetooth, Wi-Fi or DVBT) used by a primary user. Other spectrum sensing methods include wavelet transform based estimation and time-frequency analysis. A tentative classification of sensing techniques can 
be found in [8] where it is showed that the energy detection has the lowest complexity, while the matched filter has the highest complexity. Inversely, the matched filter is the most accurate detection technique, while the energy detection is the coarsest detection technique.

Next, we present a new framework for sensing configuration and implementation.

\section{NeW ARChitecture AND CONFIGURATION FOR SPECTRUM SENSING}

This section presents a novel architecture for finding spectrum opportunities while meeting the regulatory body constraints. A cooperative sensing scheme is considered where a master node defines and sends the sensing configuration parameters to a set of sensing nodes distributed randomly around the master node. The proposed architecture is for the sensing nodes. The challenge to be solved is the following: How a given sensing node can efficiently use the configuration settings in order to find spectrum opportunities while fulfilling the regulatory requirements?

\section{A. System Presentation}

The proposed system framework is depicted in Fig. 1. The sensing node is assumed to be able to perform $K$ different sensing algorithms. The algorithms could be softwareimplemented in order to optimize the circuit size. The system is comprised of a sensing process (performed more often) and a knowledge building process (occasionally performed).

The sensing process is implemented in the sensing node. For given sensing configuration settings provided by the master node (i.e., frequency band to be sensed, primary signal type, sensing duration, maximum allowed false alarm probability, minimum allowed detection probability, noise estimation time) the sensing process consists in choosing a sensing algorithm that will perform the sensing task, and in testing the regulatory conformance with regard to the current environment and the selected sensing algorithm performance.

The knowledge building process is an off-line process aiming at finding the best sensing algorithm for each configuration scheme. It also provides the required SNR to reach a target sensing performance (e.g., maximum allowed false alarm probability, minimum allowed detection probability, sensing duration) for all the available sensing algorithms. As a result, a knowledge base is fed with the selected algorithms and their required SNR for all possible configuration schemes.

The sensing process is performed dynamically in the sensing node while the knowledge building process is performed offline (and the results are used to feed the knowledge base) only if configuration settings change.

\section{B. Sensing Process}

The sensing process is the main component of the sensing task and it runs in each sensing period. The main points are as follows:

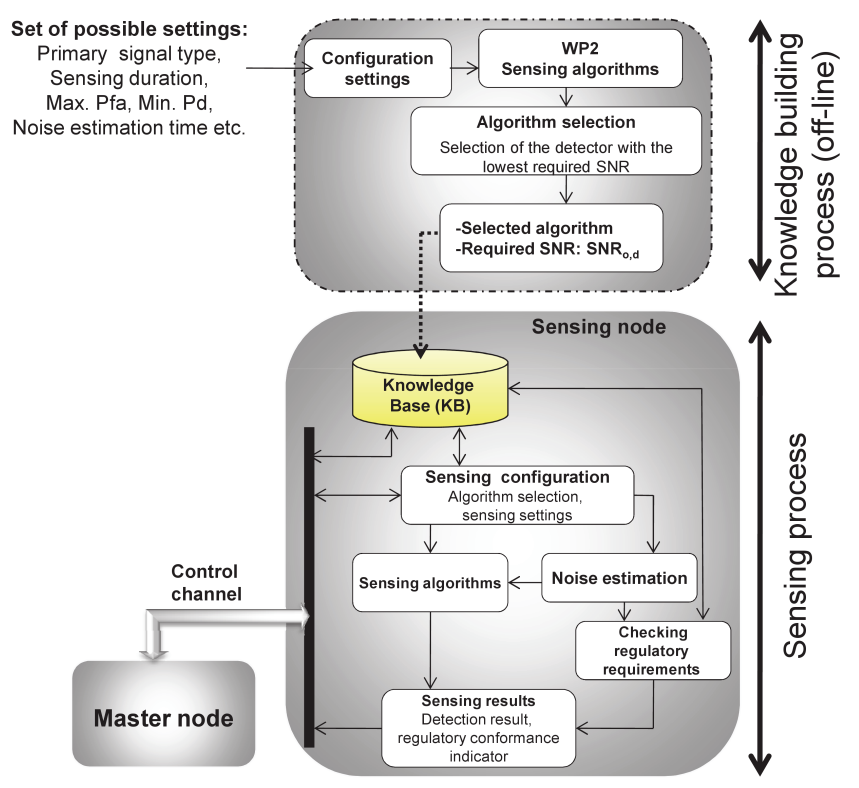

Fig. 1. System Architecture

1) Sensing Configuration Settings: the master node sends the configuration settings to the selected sensing nodes. The assumption under such a spectrum sensing scheme is that the master node is responsible of defining the overall sensing strategy and sensing scheduling. The current sensing configuration settings include the selected nodes identity, the frequency band to be sensed, the primary signal features (that are apriori known), the sensing starting time, the sensing duration, the maximum allowed false alarm probability, the minimum allowed detection probability, and the noise estimation time.

2) Sensing: the following tasks are performed.

- The sensing configuration function, of each sensing node, reads the knowledge base to get the selected algorithm corresponding to the current configuration settings.

- Noise power estimation, for the current frequency band, is performed. Noise estimation can be performed directly in the current frequency band [19], or in another frequency band assumed to have the same noise level [14], [17].

- The sensing is performed using the selected sensing algorithm (which could use the noise estimation if necessary).

- The regulatory requirement, related to the detection threshold [5], is checked for the selected detector and the current noise level. In fact, regulatory bodies (e.g., FCC) provide the detection threshold for specific primary signal. The detection threshold corresponds to the minimum received signal at which primary signal should still be accurately (e.g., with probability $P_{d \text {,min }}=90 \%$ ) detected by the CR. Therefore, using the current noise power estimation $\hat{\sigma}^{2}$, we derive the SNR required by the regulatory body to reach $P_{d, \min }$, as

$$
\mathrm{SNR}_{\mathrm{rb}}=\frac{\text { Detection threshold }}{\hat{\sigma}^{2}} .
$$

For selected detector $d$, let $\mathrm{SNR}_{0, \mathrm{~d}}$ be the required 
SNR to achieve $P_{d, \min }$ and $P_{\text {fa,max }}$ simultaneously for a given sensing duration $T_{s}$. To ensure that the current detection result conforms to the regulatory requirement, the regulatory $\mathrm{SNR}_{\mathrm{rb}}$ must be higher than the selected detector $\mathrm{SNR}_{0, \mathrm{~d}}$ (Cf. Fig. 2). Therefore, the regulatory conformance indicator, stating either $\mathrm{SNR}_{\mathrm{rb}}>\mathrm{SNR}_{0, \mathrm{~d}}$ or $\mathrm{SNR}_{\mathrm{rb}} \leq \mathrm{SNR}_{0, \mathrm{~d}}$, measures the confidence on the detection result.

- Finally, the detection result and the regulatory conformance indicator are sent to the master node that is in charge of processing the results coming from the selected nodes and also of taking the final decision about current frequency band occupancy.

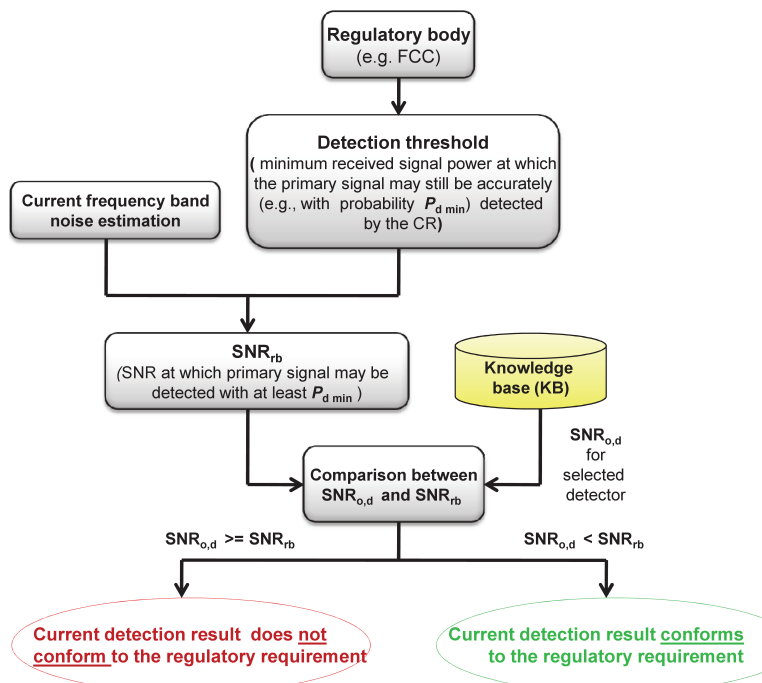

Fig. 2. Regulatory conformance checking

\section{Knowledge Building Process}

The goal of the knowledge building process is to feed the knowledge base with necessary information allowing the selection among various sensing algorithms. Moreover, the selection depends on the set of possible configuration schemes which must be known in advance. The knowledge base can be fed off-line and upgraded using an over-the-air update. The knowledge building process is depicted in Fig. 3. Given a primary signal type (e.g., DVBT, wireless microphones etc.) and a configuration scheme, we have the following steps.

- For all the available detectors, compute the mean detection probability versus the SNR.

- For each detector $d$, compute the required $\mathrm{SNR}_{0, \mathrm{~d}}$ to reach $P_{d, \text { min }}$ and $P_{f a, \max }$ simultaneously. Therefore, for selected detector $d$, when current $\mathrm{SNR}$ is greater than $\mathrm{SNR}_{0, \mathrm{~d}}$, the detection probability is greater than $P_{d, \min }$. Otherwise, the detection probability is lower than $P_{d \text {,min }}$ (Cf. Fig. 3).

- Select, as the best detector, the detector with the lowest required $\mathrm{SNR}_{0, \mathrm{~d}}$. The motivation of this selection is that, the lower $\mathrm{SNR}_{0, \mathrm{~d}}$ is, the more reliable the detector is (at least for high SNR).
- Configuration information, selected detector and $\mathrm{SNR}_{0, \mathrm{~d}}$ are stocked in the knowledge base (Cf. Fig. 4).

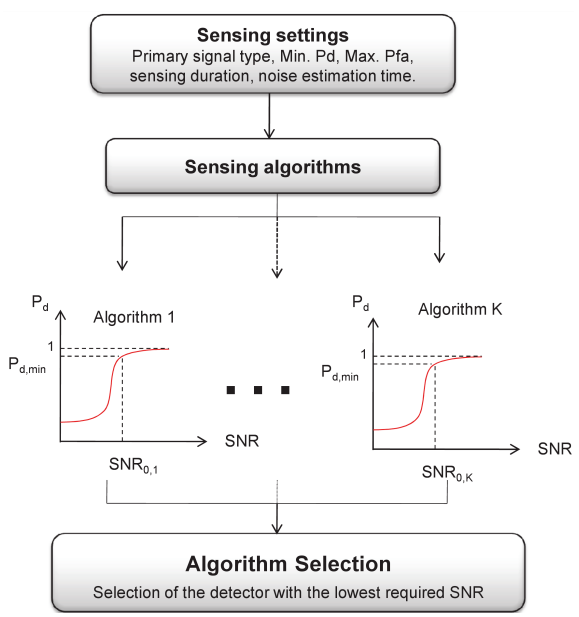

Fig. 3. Framework for the knowledge building process

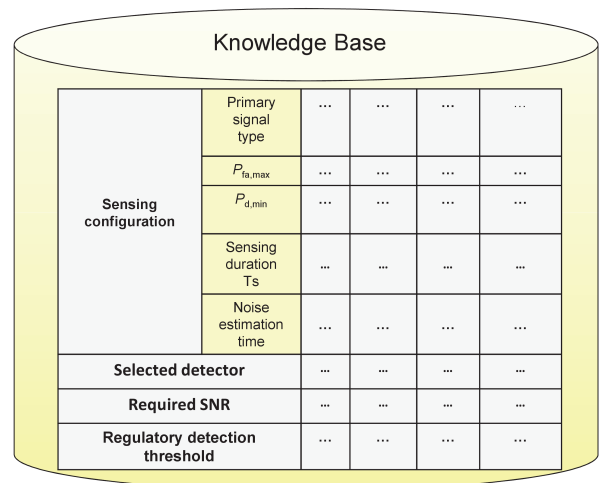

Fig. 4. Example of knowledge base for algorithm selection

\section{ASSESSMENT EXAMPLE}

In this section, we assess the above spectrum sensing architecture functionalities using two sensing algorithms: an energy detection algorithm using estimated noise variance and a cyclostationary detection algorithm called MCAS (Maximum Cyclic Autocorrelation Selection), [15].

\section{A. Energy Detection Using Estimated Noise Variance}

Due to its simplicity and its blindness regarding the signal features, the energy detector is one of the most studied sensing technique in the literature. The test statistic generated from the energy detector, over $N$ signal samples, can be written as $T=\left(\sum_{n=1}^{N} y_{n}^{2}\right) / N$. In order to distinguish between the two hypothesis of (2), the test statistic $T$ is compared with a decision threshold $\lambda$. When $N$ is sufficiently large, the probability density function of $T$ can be approximated by a Gaussian distribution. Therefore, detection probability $P_{d}$, and 
false alarm probability $P_{f a}$ can be expressed as [14]:

$$
\begin{aligned}
P_{d} & =Q\left(\sqrt{\frac{N}{2}}\left(\frac{\lambda}{\sigma^{2}(1+S N R)}-1\right)\right), \\
P_{f a} & =Q\left(\sqrt{\frac{N}{2}} \frac{\lambda-\sigma^{2}}{\sigma^{2}}\right),
\end{aligned}
$$

where SNR is the Signal-to-Noise Ratio, and $Q($.$) is the$ $\mathrm{Q}$ function [20]. From (5), the detection threshold can be obtained, given target false alarm probability $P_{f a \text {,target }}$ and noise variance $\sigma^{2}$, as $\lambda=\sigma^{2}\left(1+Q^{-1}\left(P_{f a \text {,target }}\right) / \sqrt{N / 2}\right)$. The main drawback when performing energy detection is that, in practice, the noise variance $\sigma^{2}$ is not exactly known. The total noise includes the thermal noise, the receiver noise, and the environment noise that can be time varying [14]. Therefore, the exact noise variance $\sigma^{2}$ should be replaced by an estimated noise variance $\hat{\sigma}^{2}$. The noise power level can be estimated using various techniques. Real time noise estimation can be performed in another frequency band known to be free and assumed to have the same noise level as the current sensing frequency band. In [14], the noise power is assumed to be estimated in such a free frequency band over $N$ signal samples and the real noise variance is assumed to be a realization of a Gaussian random variable with mean $\hat{\sigma}^{2}$ and variance $2 \hat{\sigma}^{4} / N$. Therefore, the detection probability and the false alarm probability are also randomly distributed. The mean false alarm probability $\bar{P}_{f a}$ (mathematical expectation over all the realizations of the noise variance) is derived in [14] as

$$
\bar{P}_{f a}=\int_{-\infty}^{+\infty} Q\left(\beta+t\left(1+\beta \sqrt{\frac{2}{N}}\right)\right) \frac{e^{-\frac{t^{2}}{2}}}{\sqrt{2 \pi}} d t
$$

where $\beta=Q^{-1}\left(P_{f a \text {,target }}\right)$. In [14], the noise is estimated over the same number of samples $N$ as the received signal $y$ is sampled over. In this paper it is assumed that the noise is estimated over $N^{\prime}$ samples acquired in a free frequency band assumed to have the same noise level as the sensing frequency band. It is therefore assumed that the noise is estimated over a time duration $T_{n}$ that may be different from the sensing duration $T_{s}$ over which the sensed signal is sampled. Under this assumption, the real noise power is a realization of a Gaussian random variable with mean $\hat{\sigma}^{2}$ and variance $2 \hat{\sigma}^{4} / N^{\prime}$. Therefore, (6) can be rewritten (using the same calculations as in [14] ) as

$$
\bar{P}_{f a}=\int_{-\infty}^{+\infty} Q\left(\beta+t \sqrt{\frac{N}{N^{\prime}}}\left(1+\beta \sqrt{\frac{2}{N}}\right)\right) \frac{e^{-\frac{t^{2}}{2}}}{\sqrt{2 \pi}} d t .
$$

For spectrum efficiency purpose, spectrum sensing should be constrained by a maximum allowed false alarm probability, $P_{f a, \max }$. In such situation, the expression of $\bar{P}_{f a}$ allows controlling the false alarm probability of the energy detector. That is, one can compute the maximum value of $P_{f a \text {,target }}$ to meet the constraint $\bar{P}_{f a} \leq P_{f a, \max }$.

\section{B. MCAS-Based Detection}

In [15], a robust spectrum sensing method against noise uncertainty and interference is proposed. Referred to as Maximum Cyclic Autocorrelation Selection (MCAS), the proposed method is based on the cyclostationary feature of primary signals. The MCAS-based detector compares the peak and non-peak values of the cyclic autocorrelation function (CAF) to detect primary signals. A non-peak value of CAF is computed at cyclic frequencies between two consecutive peaks. The desired false alarm probability is obtained by setting the number of the non-peak values. In [15], it is showed that MCAS can control the false alarm probability under noise uncertainty and interference. Furthermore, MCAS achieves closer performance to the conventional cyclostationary feature detector with much less computational complexity.

\section{Numerical Example}

We consider the two previous detection algorithms: the energy detector and the MCAS-based detector. The licensed frequency band width is set to $W=7.61 \mathrm{MHz}$ (e.g., the DVBT in Europe). The sampling frequency is set to $f_{s}=W$. The primary system uses an OFDM modulation with 1024 subcarriers, $224 \mu s$ useful symbol period and $224 / 4=56$ $\mu s$ cyclic prefix length. Sensing duration $T_{s}$, noise estimation time, $P_{d, \text { min }}$ and $P_{f a, \max }$ are set in Table I.

Fig. 5 represents the detection probability in terms of SNR for the energy detector (with noise estimation durations $5 \mathrm{~ms}$ and $5 / 30 \mathrm{~ms}$ ) and for the MCAS. One can notice that the required $\mathrm{SNR}_{0, \mathrm{~d}}$ to reach $P_{d, \min }$ and $P_{f a, \max }$ for the MCAS is equal to $-12 \mathrm{~dB}$. Further, the required $\mathrm{SNR}_{0, \mathrm{~d}}$ is equal to $-16 \mathrm{~dB}$ for the energy detector using $5 \mathrm{~ms}$ noise estimation time, and is equal to $-9.58 \mathrm{~dB}$ when noise estimation time is $5 / 30 \mathrm{~ms}$. Therefore, the energy detector is selected when noise estimation time is $5 \mathrm{~ms}$. When it is less or equal to $5 / 30 \mathrm{~ms}$, the MCAS is selected. Table I is an example of knowledge base that could be stored in the sensing node. One example of regulatory detection threshold is $-114 \mathrm{dBm}$ for ATSC digital TV signals, averaged over a $6 \mathrm{MHz}$ bandwidth, as specified in the FCC draft [5]. As stated above, to ensure that the current detection result conforms to the regulatory requirement, $\mathrm{SNR}_{\mathrm{rb}}$ (which is dynamically computed, in each sensing period, using the noise power estimation) must be higher than the $\mathrm{SNR}_{0, \mathrm{~d}}$ of the selected detector. Considering a cooperative sensing scenario, each sensing node should report to the master node its detection result together with the regulatory conformance indicator. Further, the master node should combine the decisions, while taking into account regulatory requirements.

\section{CONCLUSions}

Spectrum sensing is a promising solution allowing opportunistic spectrum access. This paper considered the case of single-node spectrum sensing. The cognitive radio is assumed to be able of performing various sensing algorithms. The novelty of this paper consists in proposing a new architecture and single-node configuration scheme for spectrum sensing. This 


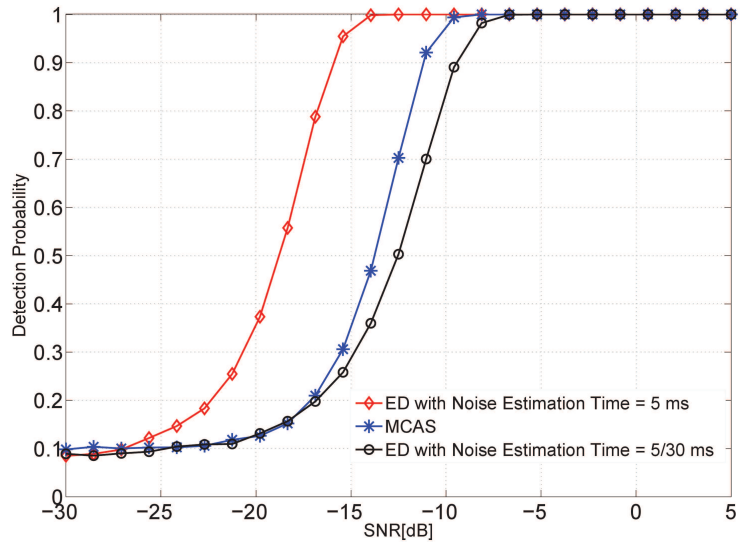

Fig. 5. Detection probability versus SNR for the MCAS and the energy detector with $5 \mathrm{~ms}$ and $(5 / 30) \mathrm{ms}$ noise estimation time.

TABLE I

KNOWLEDGE BASE FOR THE MCAS AND THE ENERGY DETECTOR (ED) WITH 5 MS AND 5/30 MS NOISE ESTIMATION TIME.

\begin{tabular}{|c|c|c|}
\hline$P_{d, \min }$ & $90 \%$ & $90 \%$ \\
\hline$P_{f a, \max }$ & $10 \%$ & $10 \%$ \\
\hline$T_{s}$ & $5 \mathrm{~ms}$ & $5 \mathrm{~ms}$ \\
\hline Noise estimation time & $5 \mathrm{~ms}$ & $5 / 30 \mathrm{~ms}$ \\
\hline Selected detector & $\mathrm{ED}$ & $\mathrm{MCAS}$ \\
\hline Required SNR & $-16 \mathrm{~dB}$ & $-12 \mathrm{~dB}$ \\
\hline Regulatory detection threshold & $-114 \mathrm{dBm}$ & $-114 \mathrm{dBm}$ \\
\hline
\end{tabular}

proposal allows a dynamic selection between the available sensing algorithms. The selection is based on a knowledge building process (that is occasionally performed), and which allows selecting the best sensing algorithm for given configuration parameters. Besides, a dynamic regulatory conformance checking is proposed: the SNR required by the regulatory body is compared to the SNR required by the current selected detector in order to reliably detect the primary signal. The proposed solution improves cooperative sensing processing and fusion. Using the proposed method, each sensing node performs the detection with the most reliable algorithm and reports to the master node the sensing result and a regulatory conformance indicator showing whether the sensing result should be assumed reliable or not.

\section{ACKNOWLEDGMENT}

The research leading to these results has received funding from the European Community's Seventh Framework Programme (FP7/2007-2013) under grant agreement SACRA number 249060 .

\section{REFERENCES}

[1] J. Mitola, "Cognitive radio: An integrated agent architecture for software defined radio", Ph.D. dissertation, KTH, Sweden, Dec. 2000.

[2] "Cisco Visual Networking Index: Global Mobile Data Traffic Forecast Update", available Online, Jan. 29, 2009.

[3] "Cisco Visual Networking Index: Global Mobile Data Traffic Forecast Update, 2009-2014”, available Online, Feb. 9, 2010.
[4] A. Shukla, A. Alptekin, J. Bradford, E. Burdidge, D. Chandler, M. Kennett, P. Levine and S. Weiss, "Cognitive Radio Technology: a Study for Ofcom”, QinetiQ, vol. 1, Feb. 2007.

[5] FCC 10-174, In the Matter of "Unlicensed Operation in the TV Broadcast Bands and Additional Spectrum for Unlicensed Devices Below 900 $\mathrm{MHz}$ and in the $3 \mathrm{GHz}$ Band ", SECOND MEMORANDUM OPINION AND ORDER, Sept. 23, 2010.

[6] D. Cabric, S. M. Mishra and R. W. Brodersen, "Implementation Issues in Spectrum Sensing for Cognitive Radio", Conference Record ThirtyEighth Asilomar Conference on Signals, Systems and Computers, vol. 1, pp. 772-776, 7-10 Nov. 2004.

[7] M. Matinmikko, T. Rauma, M. Mustonen and J. Del Ser, "Architecture and Approach for Obtaining Spectrum Availability Information ", IEEE VTC Spring, pp. 1-5, May 2011.

[8] T. Yücek and H. Arslan, "A survey of spectrum sensing algorithms for cognitive radio application", IEEE Communications Surveys and Tutorials, vol. 11, pp. 116-130, First Quarter 2009.

[9] T. Yücek and H. Arslan, "Spectrum characterization for opportunistic cognitive radio systems ", Proc. IEEE Military Commun. Conf., Washington, D.C., USA, Oct. 2006, pp. 1-6.

[10] A. Ghasemi and E. S. Sousa, "Spectrum Sensing in Cognitive Radio Networks: Requirements, Challenges and Design Trade-offs", IEEE Communications Magazine, vol. 46, pp. 32-39, Avril 2008.

[11] H. Urkowitz, "Energy detection of unknown deterministic signals", Proceedings of the IEEE, vol. 55, pp. 523-531, April 1967.

[12] A. Dandawate and G. B. Giannakis, "Statistical tests for presence of cyclostationarity", IEEE Trans. Signal Process, 42, 2355-2369 (1994).

[13] A. Ghasemi and E. S. Sousa, "Collaborative Spectrum Sensing for Opportunistic Access in Fading Environments", Proc. of IEEE DysPAN, pp. 131-136, Dec. 2005.

[14] Z. Ye, G. Memik and J. Grosspietsch, "Energy Detection using Estimated Noise Variance for Spectrum Sensing in Cognitive Radio Networks", Proc. of IEEE WCNC, pp. 711-716, March 31-April 3, 2008.

[15] K. Muraoka, M. Ariyoshi, "A Robust Spectrum Sensing Method Based On Maximum Cyclic Autocorrelation Selection for Dynamic Spectrum Access", IEICE Trans. Commun., Vol. E92-B, No.12, Dec. 2009.

[16] S. Maleki, A. Pandharipande and G. Leus, "Two-stage spectrum sensing for cognitive radios", Proc. IEEE ICASSP, pp. 2946-2949, Mar. 2010.

[17] D. Panaitopol, A. Bagayoko, P. Delahaye and L. Rakotoharison, "Fast and Reliable Sensing Using a Background Process for Noise Estimation," Proc. CrownCom, Osaka, Japan, 1-3 June, 2011.

[18] R. Tandra and A. Sahai, "Fundamental limits on detection in low SNR under noise uncertainty," Proc. of International Conference on Wireless Networks, Communications and Mobile Computing, vol. 1, pp. 464-469, 13-16 June 2005.

[19] M. J. Ready, M. L. Downey and L. J. Corbalis, "Automatic noise floor spectrum estimation in the presence of signals", Proceedings of ThirtyFirst Asilomar Conference on Signals, Systems and Computers, vol. 1, pp. 877 - 881, Nov. 1997.

[20] A. Papoulis, Probability, Random Variables, and Stochastic Processes, McGraw-Hill, 2002.

[21] J. G. Proakis, Digital Communications, 4th Ed. McGraw-Hill, 2001. 